\title{
Imaging modalities in synchronous oligometastatic prostate cancer
}

\author{
Jurgen J. Futterer ${ }^{1} \cdot$ Cristian Surcel $^{2} \cdot$ Roderick van den Bergh $^{3} \cdot$ Hendrik Borgmann $^{4} \cdot$ Alberto Briganti $^{5}$. \\ Giorgio Gandaglia ${ }^{5} \cdot$ Alexander Kretschmer $^{6} \cdot$ Piet Ost $^{7} \cdot$ Prasanna Sooriakumaran $^{8} \cdot$ Derya Tilki $^{9,13}$. \\ Massimo Valerio ${ }^{10}$. Guillaume Ploussard ${ }^{11}$. Pieter J. L. De Visschere ${ }^{12} \cdot$ Igor Tsaur $^{4}$ on behalf of the EAU-YAU \\ Prostate Cancer Working Party
}

Received: 4 April 2018 / Accepted: 18 July 2018 / Published online: 1 August 2018

(c) The Author(s) 2018

\begin{abstract}
Purpose Along with a number of other malignancies, the term "oligometastatic" prostate cancer has recently emerged. It represents an attempt to define a subtype of cancer with a limited metastatic load that might perform more favorably than a distinctly disseminated disease, or even one that may be managed in a potentially curative way. Since there is currently a knowledge gap of what imaging modalities should be utilized to classify patients as having this type of tumor, we aimed to shed light on the role of conventional and marker-based imaging in the setting of synchronous oligometastatic prostate cancer as well as summarize the available evidence for its clinical application.

Methods A literature search on December 15th 2017 was conducted using the Pubmed database.

Results Functional imaging techniques like ${ }^{68} \mathrm{Ga}$ PSMA. ${ }^{68} \mathrm{Ga}$ PSMA PET-CT has currently been shown the best detection rates for the assessment of nodal, bone and visceral metastases, especially for smaller lesions at low PSA levels.

Conclusions Functional imaging helps detect low-burden disease metastatic patients. However, these imaging modalities are not available in every center and thus clinicians may be prone to prescribe systemic treatment rather than referring patients for cytoreductive treatments. We hope that the ongoing prospective trials will help guide clinicians in making a more personalized management of synchronous metastatic patients.
\end{abstract}

Keywords Prostate cancer $\cdot$ Oligometastatic $\cdot$ Imaging $\cdot$ PET-CT $\cdot$ PSMA $\cdot$ MRI

Jurgen J. Futterer and Cristian Surcel contributed equally as first authors.

Pieter J. L. De Visschere and Igor Tsaur contributed equally as senior authors.

Jurgen J. Futterer

jurgen.futterer@radboudumc.nl

1 Department of Radiology and Nuclear Medicine, Radboudumc, P.O. Box 9101, internal postal code 766, 6500 HB Nijmegen, The Netherlands

2 Department of Urology, Fundeni Clinical Institute, Carol Davila University of Medicine, Bucharest, Romania

3 Department of Urology, University of Utrecht, Utrecht, The Netherlands

4 Department of Urology and Pediatric Urology, University Medicine Mainz, Mainz, Germany

5 Department of Urology, Urological Research Institute, Vita-Salute University and San Raffaele Hospital, Milan, Italy

6 Department of Urology, Ludwig-Maximilians-University of Munich, Munich, Germany
7 Department of Radiation Oncology and Experimental Cancer Research, Ghent University Hospital, Ghent, Belgium

8 Department of Uro-oncology, University College London Hospital, London, UK

9 Martini-Klinik Prostate Cancer Center, University Hospital Hamburg-Eppendorf, Hamburg, Germany

10 Department of Urology, Centre Hospitalier Universitaire Vaudois, Lausanne, Switzerland

11 Department of Urology, Saint Jean Languedoc Hospital, Toulouse, France

12 Department of Radiology and Nuclear Medicine, Ghent University Hospital, Ghent, Belgium

13 Department of Urology, University Hospital Hamburg-Eppendorf, Hamburg, Germany 


\section{Introduction}

A total of 416,700 new cases of prostate cancer (PCa) were estimated to occur in Europe in 2012, while 92,200 males would succumb, making PCa besides non-melanoma skin cancers the most frequently diagnosed malignancy and at the same time the third leading cause of cancer death in men [1]. In non-metastatic disease, curative risk adapted approaches incorporating life expectancy, comorbidities and treatment preference, and at the same time accounting for the presumed clinical stage of the tumor, are offered for patients to choose between active surveillance, focal therapy, radical prostatectomy (RP) or radiation therapy (RT) accompanied by excellent survival probabilities [2].

Fortunately, only less than 1 out of 20 males with PCa is nowadays diagnosed with a de novo systemically disseminated cancer due to a wide adoption of PSA screening [3]. Once metastasized, primarily or progressing after local treatment, disease is, however, believed incurable and only manageable with palliative intent. Since Huggins and Hodges determined the hormonal dependence of PCa in the 1940s [4], androgen deprivation therapy (ADT) has been the frontline treatment for metastatic disease. However, disease progression to castration-resistant $\mathrm{PCa}(\mathrm{CRPCa})$ inevitably occurs after 2-3 years of ADT [5].

In general, disease classification as "metastatic" as opposed to "clinically localized" relies on the presence of metastatic lesions on conventional imaging, i.e., lymphatic or visceral metastases on computed tomography (CT) or magnetic resonance imaging (MRI) as well as osseous metastases on bone scan in accordance with recommendations of the European Association of Urology on imaging for disease staging [6]. In the past, men with metastatic disease were usually deprived of a local treatment (and subjected to ADT) under the assumption that this therapy may not exert any positive influence on the following course of the disease and may expose patients to unnecessary treatment-related morbidity. Consequently, it was carried out solely in highly selected cases experiencing local complications. Common practice was, e.g., the abandonment of RP if pelvic lymph node dissection demonstrated lymphatic metastasis on frozen section analysis. However, evidence emerged later that patients with positive nodes and complete RP have a survival advantage over their counterparts with abandoned RP [7, 8]. Thus, a number of patients with limited extent of disseminated disease might still benefit from local treatment (RP or $\mathrm{RT}$ ), not only in terms of its alleviating local symptoms as a palliative care but also by experiencing a survival advantage (if not a cure) via a multimodal approach [9-11].

Ongoing challenge for a treating physician hence consists of identifying patients whose disease has progressed systemically, but in whom a local therapy combined with metastasis-directed and/or adjuvant systemic therapy might be possible. In this context, the term "oligometastatic disease" represents an attempt to define such a population of PCa patients. In view of a virtual revolution achieved in the last years in the area of molecular imaging modalities in $\mathrm{PCa}$ allowing for an improved sensitivity and specificity of metastasis detection in small-volume disease, it is apparent that the numbers of patients detected with a low-burden disseminated cancer as compared to localized disease will rise in the future, since occult lesions missed with conventional imaging may be better visualized with these novel methods.

Since there is neither unanimous consent on how to exactly classify this disease nor what imaging should be utilized for its definition, we strived to review the available evidence on conventional and molecular imaging modalities and their current and possible future clinical implication in the setting of the synchronous oligometastatic PCa (OPCa).

\section{Oligometastatic disease}

Oligometastasis was first described by Hellmann and Weichselbaum in 1995, as they postulated that tumor progression occurs in a multistep fashion during its clinical evolution and the number and sites of metastatic lesions may reflect the state of tumor development [12]. According to this theory, there are intermediate cancer stages between purely localized and widely metastatic. Tumors in their early state of progression possess only a low dissemination capacity and hence lead to a few lesions limited to organs with the highest host receptivity offering optimal conditions for growth, proliferation and angiogenesis and where these cells are with a fragile invasive behavior may survive. Following this, malignant seeding capacity intensifies and promotes a multifocal dissemination. The authors also proposed that some patients bearing oligometastatic disease might be treated with curative intent.

In 2004, Singh et al. [13] were the first to use the term "oligometastatic disease" in the setting of PCa. They reported that $\mathrm{PCa}$ patients, who were initially treated with RT for localized/locally advanced disease and later developed OPCa (defined as $\leq 5$ lesions; metachronous oligometastasis), had a superior survival over their counterparts with a more extended dissemination. They proposed to treat patients with OPCa aggressively to counteract further systemic seeding from metastatic lesions and improve longterm survival.

Traditionally, OPCa is referred to as a low-volume tumor burden up to 3-5 metastases in bone and/or lymph nodes on conventional imaging [3,14]. Of note, novel imaging modalities such as ${ }^{11} \mathrm{C}$-Choline, ${ }^{11} \mathrm{C}$-Acetate, ${ }^{18} \mathrm{~F}$-FDG or ${ }^{18} \mathrm{~F}$-Choline PET-CT have also been used to classify patients as having OPCa [15-18]. Importantly, it is still 
unclear whether OPCa is simply a transitory phenomenon from clinically localized to polymetastatic cancer and shares similar molecular features with a high-burden disease [14]. Alternatively, the underlying biology at the molecular level in OPCa cells might be different to polymetastatic disease. Preclinical data suggest that oligometastatic progression of tumors is substantially driven by epigenetic alterations and, in particular, miRNAs, whereas polymetastatic dissemination is characterized by overexpression of genes associated with cell division and cell cycle progression [19-21]. This scenario might also hold true for PCa. Further comprehensive research in $\mathrm{PCa}$ is urgently needed to elucidate if there are really different molecular mechanisms between the true oligometastatic and pseudo-oligometastatic disease, which has already disseminated to a considerable extent with occult lesions, to facilitate selection of patients for local and/or metastases-targeted and/or adjuvant systemic therapy vs. palliative systemic treatment [14]. In addition, molecular classifiers are welcomed to pigeonhole OPCa patients with a predominantly androgen receptor-driven disease and for whom ADT or abiraterone would be an effective treatment as an adjuvant approach as opposed to those who might progress rapidly to CRPC. In particular, there might be some histological subtypes of $\mathrm{PCa}$, such as ductal or neuroendocrine/small cell variants, which might benefit most from chemotherapy even in the oligometastatic state [22].

\section{CT and MRI}

Both CT and MRI are the mainstay of whole-body morphological imaging; functional techniques in MRI provide additive data [23]. The imaging depicted metastatic state is key to patient management for biomarker development and for therapeutic clinical trials [24]. The literature shows a broad range in the diagnostic performance of both unenhanced CT and MRI in the detection of lymph node metastases. CT and MRI demonstrate an equally poor performance in the detection of lymph node metastases from PCa. Reliance on either CT or MRI will misrepresent the patient's true status regarding nodal metastases, and thus misdirect the therapeutic strategies offered to the patient [25].

Whole-body MRI (WB-MRI) with conventional T1, T2 and inversion recovery sequences provides high tissue contrast for bone metastasis detection [26, 27]. The addition of diffusion-weighted imaging (DWI) to WB-MRI enables the study of extra-skeletal involvement, including lymph nodes and other soft-tissue metastases, without requiring intravenous contrast agents $[28,29]$. The reported sensitivity and specificity for WB-MRI ranges from 98 to $100 \%$ and 98 to $100 \%$, respectively. Inter-observer agreement for reading of WB-MRI images that include DW is very good $(K=0.87$
[0.66; 1.00]) [27]. Furthermore, DWI in WB-MRI will allow quantification of tumor load [30]. The METastasis Reporting and Data System for Prostate Cancer (MET-RADS-P) imaging recommendations are designed to promote standardization and diminish variations in the acquisition, interpretation, and reporting of WB-MRI in advanced prostate cancer. The MET-RADS-P system provides comprehensive characterization of advanced PCa state, not only at the start of treatments but also over time as the disease evolves [30] (Fig. 1).

\section{Bone scan}

For the staging of bone metastases, ${ }^{99 \mathrm{~m}}$ Technetium-methylene diphosphonate bone scintigraphy ('bone scan') is the traditional established imaging modality. In patients with primary $\mathrm{PCa}$, the current guidelines recommend a bone scan if there are symptoms suggestive of bone metastasis, or in asymptomatic patients when the PSA level is $>10 \mathrm{ng} / \mathrm{ml}$, Gleason score $\geq 8$ or clinical stage $\geq \mathrm{T} 3$ (i.e., intermediateand high-risk tumors) [6]. Bone scan has the disadvantage that it only assesses the skeleton and that it is hampered by a large number of inconclusive results warranting additional imaging with radiography, CT or MRI in about $16 \%$ of the cases $[31,32]$. A bone scan can readily detect polymetastatic disease in symptomatic patients or in patients with PSA levels of $\geq 50 \mathrm{ng} / \mathrm{ml}$ with reported sensitivities of 78-95\%, specificities of $72-100 \%$, positive predictive value of $96 \%$ and negative predictive value of $60 \%[32,33]$. In asymptomatic patients the diagnostic yield is highly dependent on the PSA level, with positivity rates of only $2.3 \%$ for PSA levels of $0-9.9 \mathrm{ng} / \mathrm{ml}, 5.3 \%$ for PSA levels of $10-19.9 \mathrm{ng} / \mathrm{ml}$ and $16.2 \%$ for PSA levels of 20-49.9 $\mathrm{ng} / \mathrm{ml}$ [34]. In recent years, with the advent of whole-body MRI and PET-CT as new comparators, the true overall sensitivity of bone scanning has appeared to be only around $65 \%$, suggesting that a significant amount of metastatic lesions may go undetected $[27,35]$. Bone scan thus seems to systematically underestimate the burden of bone metastases in the primary staging of $\mathrm{PCa}$ and consequently performs insufficiently to reliably classify patients as oligometastatic $[23,35]$.

\section{PET-CT and PET-MRI}

Recent developments in molecular imaging, in particular positron emission tomography (PET) using targeted PCa tracers, have demonstrated great promise in more accurate assessment of patients with oligometastatic disease [36]. The combination of metabolic PET imaging with morphological imaging in a hybrid PET-CT or PET-MRI exam has overcome the limitations of conventional imaging, particularly 

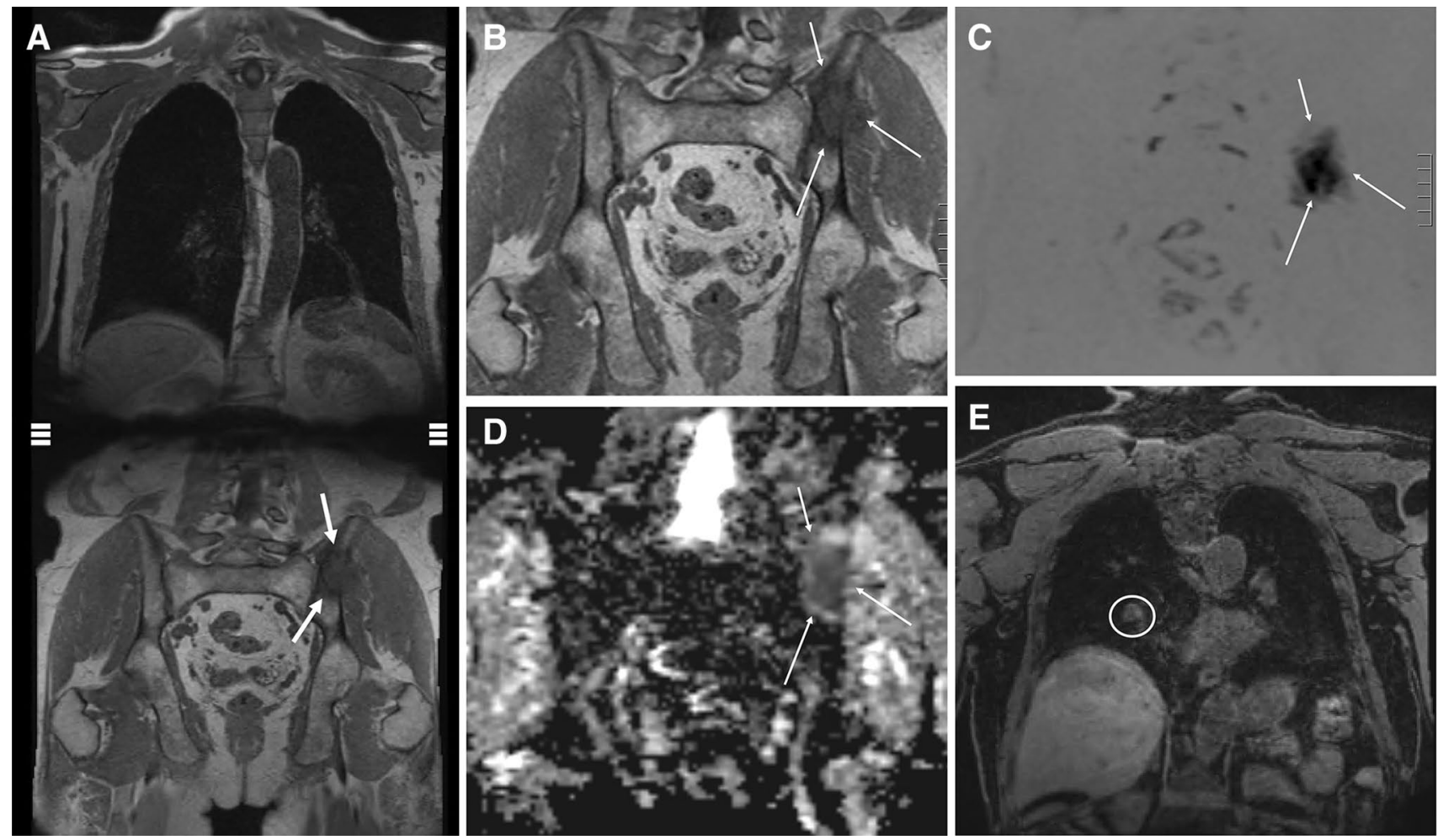

Fig. 1 73-year-old man with a Gleason $4+3$ PCa pT3 with PSA of $18.2 \mathrm{ng} / \mathrm{ml}$. A whole-body MR examination was performed. Coronal whole body T1-weighted image (a) shows a left iliac bone metastasis

in the early detection of a limited number of metastases and in the setting of low PSA levels [35, 37]. Advantages of PET-MRI over PET-CT include less exposure to radiation and better correlation of a hot spot on PET with the intraprostatic lesion or changes in the bone marrow, but there is still poor clinical evidence of PET-MRI at this time [38]. In PCa, PET-CT has been investigated mainly for restaging of patients with biochemical recurrence after treatment but there is a growing body of evidence supporting its use in primary staging $[35,39,40]$.

${ }^{18} \mathrm{~F}$ Fluoro-deoxy-glucose (FDG)-PET is the mainstay of molecular imaging and has demonstrated clinical usefulness in many tumors but has proven to be of limited value in $\mathrm{PCa}$ [31, 41]. Radiopharmaceutical agents showing a tropism for $\mathrm{PCa}$ cells such as ${ }^{11} \mathrm{C}$ acetate, ${ }^{11} \mathrm{C}$ choline and ${ }^{18} \mathrm{~F}$ choline have been introduced around the turn of the century, and, in the last 10 years predominantly choline-based tracers have been used in clinical practice [41-44]. While the early results were promising, the utility of choline PET-CT has been limited in the oligometastatic setting due to reduced sensitivity in patients with low PSA and in the early stages of metastatic spread [37]. Moreover, choline is not necessarily cancer-specific and it shows also uptake in areas of benign inflammation causing false-positive results in, for example, reactive lymph nodes [35]. The most promising (b; magnification; arrows). The bone metastasis (arrows) are evident on whole-body DWI images (c, d). e In the right lung, para-hilar, a $15 \mathrm{~mm}$ metastasis was observed (circle)

data to date have been generated with radiotracers targeting the prostate-specific membrane antigen (PSMA) which is overexpressed in PCa cells $[35,45]$. Initially reported in $2012,{ }^{68} \mathrm{Ga}$ PSMA HBED-CC PET-CT has shown to be superior to the older tracers predominantly owing to increased avidity of uptake and higher target-to-background ratio, resulting in an improved detection rate $[45,46]$. Other emerging innovative PET radiotracers for $\mathrm{PCa}$ have been proposed such as ${ }^{18} \mathrm{~F}$-Fluciclovine (FACBC), ${ }^{18} \mathrm{~F}$-Bombesin, ${ }^{18} \mathrm{~F}$-fluoro- $5 \alpha$-dihydrotestosterone (FDHT) and urokinasetype plasminogen activator receptor (UPAR), but these are almost exclusively being investigated in the setting of recurrent $\mathrm{PCa}$ or for visualization of the primary intraprostatic lesion but not for distant staging in primary $\mathrm{PCa}$ [41].

Regarding nodal staging in the primary setting, an extended pelvic lymphadenectomy still represents the gold standard and accurate imaging modalities are currently lacking. Metastatic lymph nodal spread is present in approximately $20-35 \%$ of the intermediate- and high-risk patients but they are the most common site of occult disease in de novo settings [46-48]. PET-CT has been shown to be superior as compared to conventional morphological CT or MRI in preoperative lymph node assessment $[25,49]$. PET-CT using ${ }^{11} \mathrm{C}$ choline or ${ }^{18} \mathrm{~F}$ choline tracers have shown a relatively good specificity of $89.5-99.7 \%$ and positive predictive 
value of $63.6-98.0 \%$ but only moderate sensitivity (ranging from 10 to $67 \%$ ) for detecting involved lymph nodes [39, 41, 44, 49-54]. The sensitivity is higher in high-risk PCa patients (83.3\%) than in the intermediate-risk group (33.3\%) [49]. Choline PET-CT could thus provide helpful additional information in patients with suspected oligometastatic disease but since sufficient detection rates are not reached as compared to surgical lymph node dissection, its routine clinical use is not recommended at present to detect occult lymph node metastases in all patients with high-risk PCa in whom CT scan findings are normal $[6,50,52,53,55]$.

In addition, for the staging of bone metastasis, PET-CT appears to perform better than bone scan because tracer uptake may be detected in early bone marrow infiltration before osteoblastic reaction occurs (Fig. 2). A meta-analysis evaluating choline PET-CT in the diagnosis of bone involvement in intermediate or high-risk primary PCa patients showed a pooled sensitivity of $49 \%$ and a pooled specificity of $95 \%$ [31, 39, 51]. The poor sensitivity is largely due to non-recognition of early bone metastases, although choline or fluoride PET-CT detect bone metastases in up to $56 \%$ of patients with a negative or inconclusive bone scan [55, 56]. Nevertheless, because of the similar sensitivity, choline PET-CT should not replace bone scan to date.

${ }^{68} \mathrm{Ga}$ PSMA PET-CT has currently been shown the best detection rates for the assessment of nodal, bone and visceral metastases, especially for smaller lesions at low PSA levels [37]. Gupta et al. [46] reported that in patients with newly diagnosed PC and high clinical suspicion of metastases (Gleason score 9-10 and/or serum PSA $>20 \mathrm{ng} / \mathrm{ml}$ ) but nil or $\leq 3$ metastases on conventional imaging (CT and bone scan) at least 1 lesion was detected in $100 \%$ of PSMA PET-CT. ${ }^{68} \mathrm{Ga}$ PSMA PET-CT in primary staging found bone metastasis in $13 \%$ of asymptomatic patients with PSA $<10 \mathrm{ng} / \mathrm{ml}$, although these patients would normally not have a bone scan in routine clinical practice [46]. In the systematic review of Perera [40] the pooled percentage of positive ${ }^{68} \mathrm{Ga}$ PSMA PET-CT was $40 \%$ (95\% confidence interval 19-64\%) in the primary staging. Of all studies, $24 \%$ showed only oligometastases $(\leq 3)$, and $62.5 \%$ of these concerned a solitary lesion. Moreover, a high rate of incidental extra-pelvic disease (41\%) was observed [46]. Recently, another systematic review on the currently available data on ${ }^{68} \mathrm{Ga}$ PSMA PET-CT in the primary staging of high-risk PCa was published [57]. A high variation in sensitivity (range 33-99\%) was seen across the studies but with consistently high specificity (>90\%). Twelve studies were included but only five could be classified as high quality because they correlated imaging findings with histopathology. The lack of histologic correlation with positive (and indeed negative) PSMA PET-CT results makes it unclear as to the true accuracy of this novel imaging modality.
Nonetheless, preoperative staging with ${ }^{68} \mathrm{Ga}$ PSMA PETCT appears to allow for more complete and accurate primary staging of PCa patients compared to standard routine imaging and may demonstrate a large number of otherwise unknown metastatic lesions, but its clinical role is yet to be definitively determined [40]. PET-CT has been reported to alter treatment plans in $29-76 \%$ of patients as compared to staging with conventional imaging [37, 39, 49, 56]. A large proportion of the additionally detected metastatic lesions are oligometastatic and in the future, with increasing use of ${ }^{68} \mathrm{Ga}$ PSMA PET-CT, we may expect to identify a substantial higher number of patients with oligometastasis at initial presentation (Fig. 3). The clinical impact and cost effectiveness of potentially replacing current standard imaging for ${ }^{68} \mathrm{Ga}$ PSMA PET-CT in the primary setting needs, therefore, to be confirmed by prospective studies with oncological outcomes, but despite this current lack of evidence, ${ }^{68} \mathrm{Ga}$ PSMA PETC-CT seems to be increasingly performed in clinical practice in countries where it is available [37].

\section{Clinical implications}

The rationale for using local treatment, as the first step in the management of de novo metastatic $\mathrm{PCa}$, such as radical prostatectomy and radiotherapy is based on: a potential benefit in terms of improved local control, removal of the primary source for metastasis and (possibly) improved response to systemic treatments [58]. To this day, only retrospective population-based data, from different cancer registries such as Surveillance, Epidemiology, and End Results (SEER), National Cancer Database and several institutional data, support these assumptions. Despite the lack of level 1 evidence, approximately $75 \%$ of the panelists from the Advanced Prostate Cancer Consensus Conference (APCCC) 2017 agreed that there is a need for some form of local treatment in men with oligometastatic disease [59].

Although the results confirm the feasibility of cytoreductive RP, it is still unclear who would benefit from resection of the primary tumor. The ideal candidate might be a fit patient with $\leq \mathrm{T} 3$ stage, Gleason score $\leq 7$, low-volume metastatic disease, no visceral metastases and a low PSA nadir after 6 months of systemic therapy. Furthermore, imaging might be of use for selecting these candidates. The ongoing prospective randomized trials such as TRoMBone (ISRCTN15704862) and g-RAMPP (NCT02454543) will provide level 1 evidence regarding the role of surgery in patients with synchronous oligometastatic disease [60, 61].

A clinical decision based on the available data should be taken with caution due to significant limitations of these studies and unknown confounders: limited to no information regarding the associated comorbidities, different definitions of the oligometastatic state, different endpoints and 


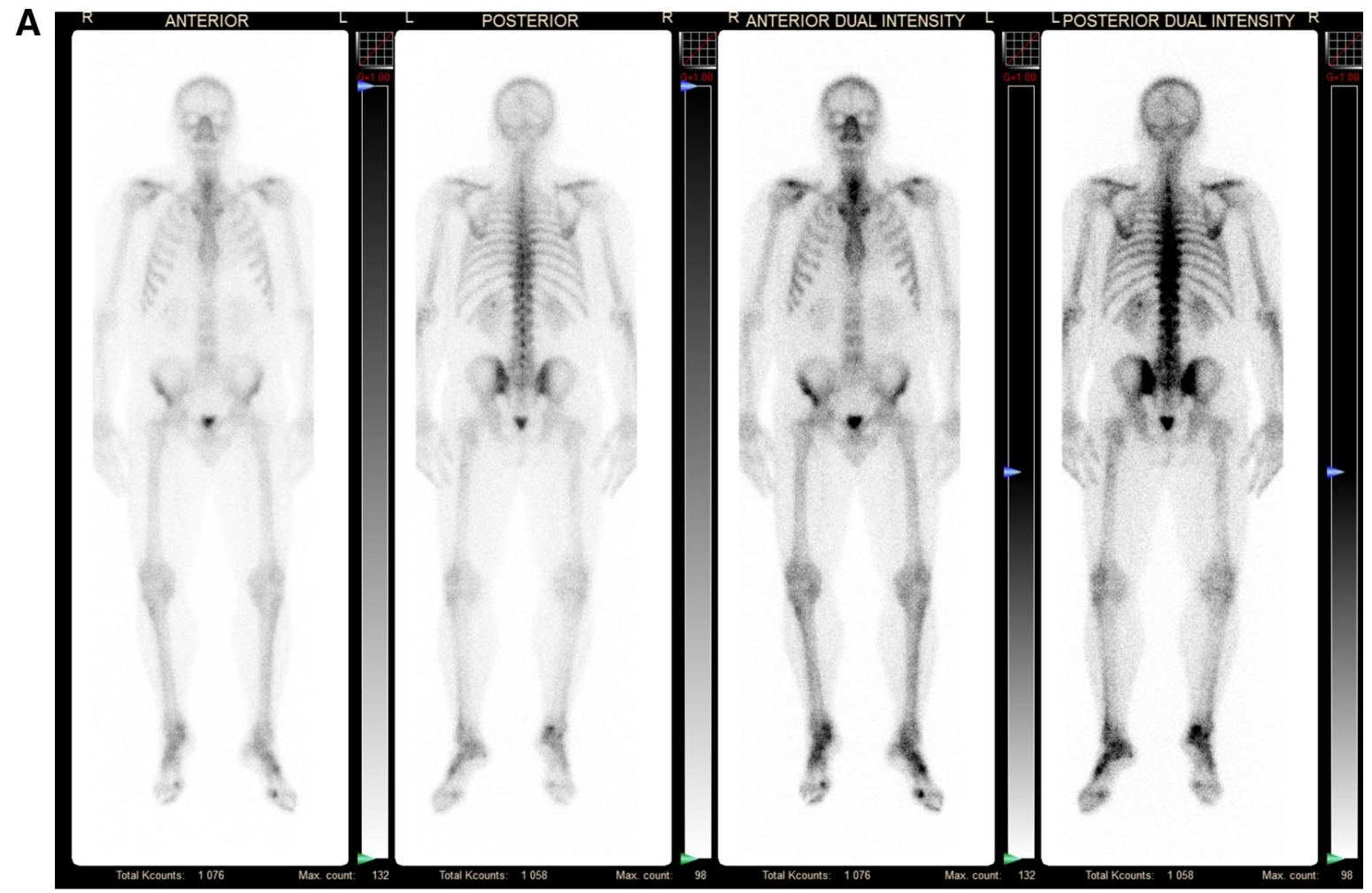

B

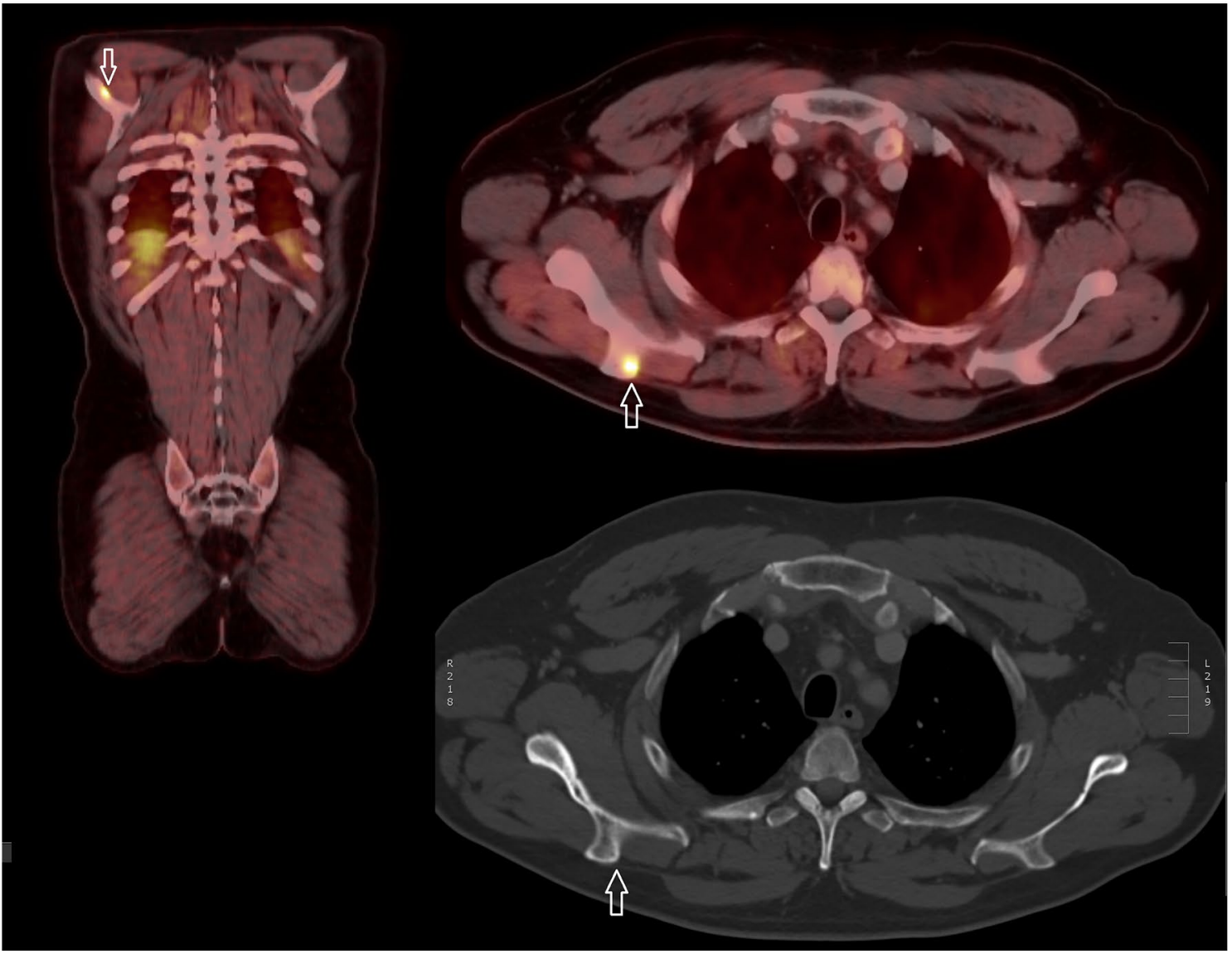


4 Fig. 2 53-year-old man with a Gleason $4+4$ PCa pT3 with PSA of $12.5 \mathrm{ng} / \mathrm{ml}$. Primary staging with bone scan showed no lesions suspicious for bone metastases (a), but because he complained of right shoulder pain a ${ }^{18} \mathrm{~F}$ choline PET-CT was performed. This demonstrated focal tracer uptake in the spina scapulae (b, white arrow), indicating a bone metastase. On the corresponding CT image the scapula was unremarkable. Choline PET-CT may demonstrate bone metastases that are occult on bone scan and CT because it can detect changes in the bone marrow before osteoblastic reaction occurs. Nevertheless, bone scan currently remains the standard imaging modality in the primary staging of $\mathrm{PCa}$

unavailable data regarding the imaging techniques used for detection of distant lesions. Furthermore, data regarding treatments such as ADT, chemotherapy or dosage of radiotherapy are largely unavailable.

\section{Role of metastasis-directed therapies}

Technical improvements in surgery and radiotherapy have introduced the option of metastasis-directed ablative therapies as an adjunct or alternative to standard-of-care systemic therapies [23]. More sensitive imaging will increase the likelihood of the detection of metastatic disease. However, none of the historic trials have incorporated any of these novel imaging modalities.

In a study by Sterzing et al., [62] 9 out of the 15 patients identified for primary staging by ${ }^{68} \mathrm{Ga}$-PSMA PET, were found to have metastatic lesions. Based on the results, approximately $51 \%$ of patients received a different therapeutic approach compared to the initial plan based upon conventional staging methods. Eight out of 15 patients were changed from M0 to M1a disease, which resulted in enlarged lymphatic field irradiation. These patients were all included in the historical trials as conventional imaging M0-status, whereas that with the current novel imaging techniques that the same number of patients was N1/M1 on novel imaging. Nevertheless, the disease control rates in these trials are already high $[63,64]$. This begs the question whether change in management should be based on this novel imaging outside a trial as we have little evidence that metastasis-directed therapy influences a major endpoint in this setting.

\section{Future perspectives}

In absence of more robust evidence from clinical trials, it is critical to be aware of how increasing availability and practical utilization of marker-based imaging in supposed non-metastatic disease, particularly PSMA PET-CT, with all its diagnostic accuracy may change treatment paradigms and de facto make decision-making process notably delicate nowadays, not least due to patients` wish. Since patients diagnosed with PCa increasingly obtain information on their disease on the Web, it is encouraging that quality, accessibility, and usability of the respective websites has been rated high in a contemporary analysis [65]. However, promising data on PSMA PET-CT exhibited there, are not ultimately accompanied by correct indications boosting demands for this imaging modality by laypersons even in disease settings without guideline recommendation for its use. The practice of performing PSMA PET-CT as primary staging in patients with high-risk localized or locally advanced disease has already become a reality in some scenarios. These patients would be treated with radical prostatectomy ( \pm adjuvant radiotherapy) or primary radiotherapy + ADT based on the findings on conventional imaging (bone scan and CT) according to the EAU guidelines [2] but using PSMA PET-CT we would diagnose occult OPCa in some of them. Emerging dilemma is how this finding would influence our treatment strategy and how the cut-off for performing or not local treatment should be defined? Would we deprive patients of efficient local therapy eventually combined with currently still low-evidenced metastasis-directed therapy replacing it by escalated systemic treatment? Of note, long-term survival analysis of the CHAARTED Trial demonstrated a trend for patients with low-volume disease to perform even worse on chemohormonal treatment than on ADT (4\% more deaths, $\mathrm{p}=0.86$ ) [66]. On the other hand, using PSMA PET-CT in some OPCa patients (as defined by conventional imaging) would screen out those with falsepositive results, e.g., benign findings as bone hemangiomas, potentially shifting them to the cohort with clinically localized disease and enabling primary curative therapy. Until these issues are not sufficiently clarified by the results of solid clinical trials, staging of primary Pca in routine clinical practice should still be performed with conventional imaging (bone scan and CT), although we are aware of the limitations. In institutions where PSMA PET-CT is available, it could be used additionally in cases of suspected OPCa to confirm the limited number of metastatic lesions but then we are left to rely on empirical decisions and treatment strategy remains challenging.

\section{Conclusion}

Ultimately, the decision regarding when and how to treat men with OPCa requires an assessment of the risks of burden of therapy versus risks of death derived from factors such as disease progression, associated comorbidities, metastatic load and access to medical facilities. With the recent data from the CHAARTED [67] and LATITUDE trials [68], an interesting question arises: is conventional imaging enough to accurately stage a patient in modern era? 


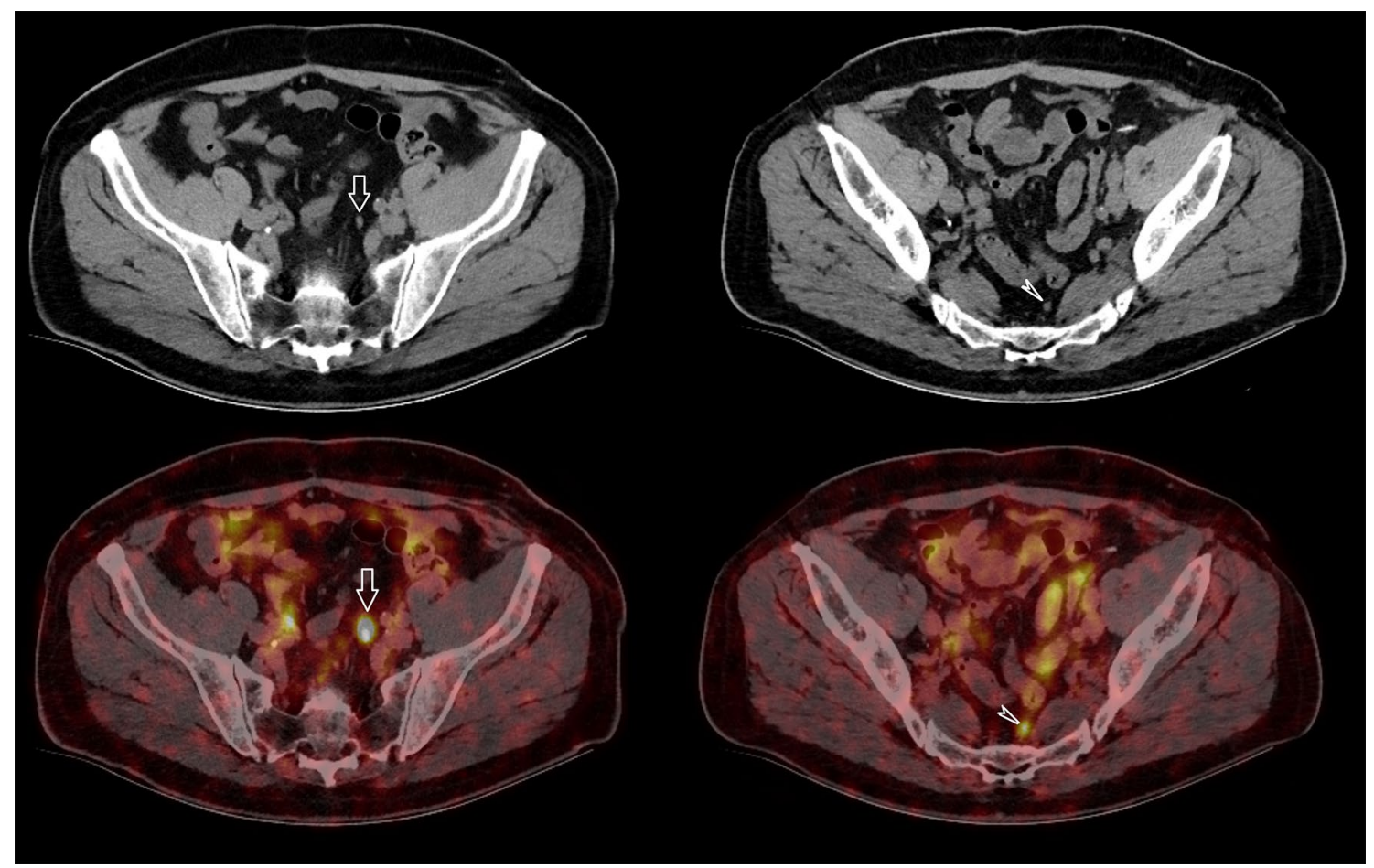

Fig. 3 72-year-old man with biopsy proven PCa Gleason $4+3$ pT2c and PSA of $6.7 \mathrm{ng} / \mathrm{ml}$. Preoperative staging with imaging revealed two small lymph nodes in the pelvis, along the left common iliac artery (white arrow) and in the presacral fat (white arrowhead). On CT scan they both have a size of $5 \mathrm{~mm}$ which is below the morphological threshold to classify them as suspicious, but on ${ }^{68} \mathrm{Ga}$ PSMA PET-CT they both showed highly avid tracer uptake, indicative of

Functional imaging helps detect low-burden disease metastatic patients who are not likely to benefit from chemotherapy. However, these imaging modalities are not available in every center and thus clinicians may be prone to prescribe systemic treatment rather than referring patients for cytoreductive treatments. We hope that the ongoing prospective trials will help guide clinicians into making a more personalized management of synchronous metastatic patients.

Author's contribution JJF: project development, data collection, manuscript writing, CS: project development, data collection, manuscript writing, RB: manuscript writing, HB: manuscript writing, AB: manuscript writing, GG: manuscript writing, AK: manuscript writing, PO: manuscript writing, PS: manuscript writing, DT: manuscript writing, MV: manuscript writing, GP: manuscript writing, PV: project development, data collection, manuscript writing, IT: project development, data collection, manuscript writing.

\section{Funding No funding}

\section{Compliance with ethical standards}

Conflict of interest The authors declare that they have no conflict of interest. lymph node metastases. Pelvic lymph node dissection was performed and both lymph nodes proved to be malignant. Preoperative staging with ${ }^{68} \mathrm{Ga}$ PSMA PET-CT thus appears to allow for more complete and accurate primary staging of PCa patients compared to standard routine imaging but its role in routine clinical practice is yet to be defined

Ethical approval/human and animal rights statement This research did not involve human participants and/or animals, but is a review paper.

Informed consent No written informed consent was obtained because this is a review paper.

Open Access This article is distributed under the terms of the Creative Commons Attribution 4.0 International License (http://creativeco mmons.org/licenses/by/4.0/), which permits unrestricted use, distribution, and reproduction in any medium, provided you give appropriate credit to the original author(s) and the source, provide a link to the Creative Commons license, and indicate if changes were made.

\section{References}

1. Ferlay J, Steliarova-Foucher E, Lortet-Tieulent J, Rosso S, Coebergh JW, Comber H et al (2013) Cancer incidence and mortality patterns in Europe: estimates for 40 countries in 2012. Eur J Cancer 49(6): 1374-1403

2. Mottet N, Bellmunt J, Bolla M, Briers E, Cumberbatch MG, De Santis M et al (2017) EAU-ESTRO-SIOG guidelines on prostate cancer. Part 1: screening, diagnosis, and local treatment with curative intent. Eur Urol 71(4):618-629 
3. Ahmad AE, Leao R, Hamilton RJ (2017) Radical prostatectomy for patients with oligometastatic prostate cancer. Oncology (Williston Park) 31(11):794-802

4. Huggins C, Hodges CV (2002) Studies on prostatic cancer: I. the effect of castration, of estrogen and of androgen injection on serum phosphatases in metastatic carcinoma of the prostate. 1941. J Urol 168(1):9-12

5. Lorente D, De Bono JS (2014) Molecular alterations and emerging targets in castration resistant prostate cancer. Eur J Cancer 50(4):753-764

6. Heidenreich A, Bastian PJ, Bellmunt J, Bolla M, Joniau S, van der Kwast $\mathrm{T}$ et al (2014) EAU guidelines on prostate cancer. Part 1: screening, diagnosis, and local treatment with curative intentupdate 2013. Eur Urol 65(1):124-137

7. Engel J, Bastian PJ, Baur H, Beer V, Chaussy C, Gschwend JE et al (2010) Survival benefit of radical prostatectomy in lymph nodepositive patients with prostate cancer. Eur Urol 57(5):754-761

8. Steuber T, Budaus L, Walz J, Zorn KC, Schlomm T, Chun F et al (2011) Radical prostatectomy improves progression-free and cancer-specific survival in men with lymph node positive prostate cancer in the prostate-specific antigen era: a confirmatory study. BJU Int 107(11):1755-1761

9. Gakis G, Boorjian SA, Briganti A, Joniau S, Karazanashvili G, Karnes RJ et al (2014) The role of radical prostatectomy and lymph node dissection in lymph node-positive prostate cancer: a systematic review of the literature. Eur Urol 66(2):191-199

10. Da Pozzo LF, Cozzarini C, Briganti A, Suardi N, Salonia A, Bertini R et al (2009) Long-term follow-up of patients with prostate cancer and nodal metastases treated by pelvic lymphadenectomy and radical prostatectomy: the positive impact of adjuvant radiotherapy. Eur Urol 55(5):1003-1011

11. Mitin T, Blute M, Lee R, Efstathiou J (2013) Management of lymph node-positive prostate cancer: the role of surgery and radiation therapy. Oncology (Williston Park) 27(7):647-655

12. Hellman S, Weichselbaum RR (1995) Oligometastases. J Clin Oncol 13(1):8-10

13. Singh D, Yi WS, Brasacchio RA, Muhs AG, Smudzin T, Williams JP et al (2004) Is there a favorable subset of patients with prostate cancer who develop oligometastases? Int J Radiat Oncol Biol Phys 58(1):3-10

14. Sonpavde G (2017) The biology of prostate cancer metastases: does oligo differ from polymetastatic? Curr Opin Urol 27(6):542-546

15. Decaestecker K, De Meerleer G, Lambert B, Delrue L, Fonteyne $\mathrm{V}$, Claeys $\mathrm{T}$ et al (2014) Repeated stereotactic body radiotherapy for oligometastatic prostate cancer recurrence. Radiat Oncol 9:135

16. Schick U, Jorcano S, Nouet P, Rouzaud M, Vees H, Zilli T et al (2013) Androgen deprivation and high-dose radiotherapy for oligometastatic prostate cancer patients with less than five regional and/or distant metastases. Acta Oncol 52(8):1622-1628

17. Ahmed KA, Barney BM, Davis BJ, Park SS, Kwon ED, Olivier KR (2012) Stereotactic body radiation therapy in the treatment of oligometastatic prostate cancer. Front Oncol 2:215

18. Berkovic P, De Meerleer G, Delrue L, Lambert B, Fonteyne V, Lumen N et al (2013) Salvage stereotactic body radiotherapy for patients with limited prostate cancer metastases: deferring androgen deprivation therapy. Clin Genitourin Cancer 11(1):27-32

19. Uppal A, Ferguson MK, Posner MC, Hellman S, Khodarev NN, Weichselbaum RR (2014) Towards a molecular basis of oligometastatic disease: potential role of micro-RNAs. Clin Exp Metastasis 31(6):735-748

20. Lussier YA, Khodarev NN, Regan K, Corbin K, Li H, Ganai $\mathrm{S}$ et al (2012) Oligo- and polymetastatic progression in lung metastasis(es) patients is associated with specific microRNAs. PLoS ONE 7(12):e50141
21. Wuttig D, Baier B, Fuessel S, Meinhardt M, Herr A, Hoefling C et al (2009) Gene signatures of pulmonary metastases of renal cell carcinoma reflect the disease-free interval and the number of metastases per patient. Int J Cancer 125(2):474-482

22. Dorff TB, Sweeney CJ (2017) Chemotherapy for oligometastatic prostate cancer. Curr Opin Urol 27(6):553-558

23. deSouza NM, Liu Y, Chiti A, Oprea-Lager D, Gebhart G, Van Beers BE et al (2018) Strategies and technical challenges for imaging oligometastatic disease: recommendations from the European Organisation for Research and Treatment of Cancer imaging group. Eur J Cancer. https://doi.org/10.1016/j.ejca.2017.12.012

24. Scher HI, Morris MJ, Stadler WM, Higano C, Basch E, Fizazi $\mathrm{K}$ et al (2016) Trial design and objectives for castration-resistant prostate cancer: updated recommendations from the Prostate Cancer Clinical Trials Working Group 3. J Clin Oncol 34(12):1402-1418

25. Hovels AM, Heesakkers RA, Adang EM, Jager GJ, Strum S, Hoogeveen YL et al (2008) The diagnostic accuracy of CT and MRI in the staging of pelvic lymph nodes in patients with prostate cancer: a meta-analysis. Clin Radiol 63(4):387-395

26. Nakanishi K, Kobayashi M, Takahashi S, Nakata S, Kyakuno M, Nakaguchi K et al (2005) Whole body MRI for detecting metastatic bone tumor: comparison with bone scintigrams. Magn Reson Med Sci 4(1):11-17

27. Lecouvet FE, El Mouedden J, Collette L, Coche E, Danse E, Jamar F et al (2012) Can whole-body magnetic resonance imaging with diffusion-weighted imaging replace Tc $99 \mathrm{~m}$ bone scanning and computed tomography for single-step detection of metastases in patients with high-risk prostate cancer? Eur Urol 62(1):68-75

28. Koh DM, Takahara T, Imai Y, Collins DJ (2007) Practical aspects of assessing tumors using clinical diffusion-weighted imaging in the body. Magn Reson Med Sci 6(4):211-224

29. Venkitaraman R, Cook GJ, Dearnaley DP, Parker CC, Khoo V, Eeles R et al (2009) Whole-body magnetic resonance imaging in the detection of skeletal metastases in patients with prostate cancer. J Med Imaging Radiat Oncol 53(3):241-247

30. Padhani AR, Lecouvet FE, Tunariu N, Koh DM, De Keyzer F, Collins DJ et al (2017) METastasis reporting and data system for prostate cancer: practical guidelines for acquisition, interpretation, and reporting of whole-body magnetic resonance imaging-based evaluations of multiorgan involvement in advanced prostate cancer. Eur Urol 71(1):81-92

31. Picchio M, Spinapolice EG, Fallanca F, Crivellaro C, Giovacchini G, Gianolli L et al (2012) $\left[{ }^{11} \mathrm{C}\right]$ Choline PET/CT detection of bone metastases in patients with PSA progression after primary treatment for prostate cancer: comparison with bone scintigraphy. Eur J Nucl Med Mol Imaging 39(1):13-26

32. Wondergem M, van der Zant FM, Knol RJJ, Burgers AMG, Bos SD, de Jong IJ et al (2018) (99m)Tc-HDP bone scintigraphy and (18)F-sodiumfluoride PET/CT in primary staging of patients with prostate cancer. World J Urol 36(1):27-34

33. Fonager RF, Zacho HD, Langkilde NC, Fledelius J, Ejlersen JA, Haarmark C et al (2017) Diagnostic test accuracy study of (18) F-sodium fluoride PET/CT, (99m)Tc-labelled diphosphonate SPECT/CT, and planar bone scintigraphy for diagnosis of bone metastases in newly diagnosed, high-risk prostate cancer. Am J Nucl Med Mol Imaging 7(5):218-227

34. Abuzallouf S, Dayes I, Lukka H (2004) Baseline staging of newly diagnosed prostate cancer: a summary of the literature. $\mathrm{J}$ Urol 171(6 Pt 1):2122-2127

35. Joice GA, Rowe SP, Pienta KJ, Gorin MA (2017) Oligometastatic prostate cancer: shaping the definition with molecular 
imaging and an improved understanding of tumor biology. Curr Opin Urol 27(6):533-541

36. Murphy DG, Sweeney CJ, Tombal B (2017) "Gotta Catch "em All", or do we? Pokemet Approach to Metastatic Prostate Cancer. Eur Urol 72(1):1-3

37. Udovicich C, Perera M, Hofman MS, Siva S, Del Rio A, Murphy DG et al (2017) (68)Ga-prostate-specific membrane antigen-positron emission tomography/computed tomography in advanced prostate cancer: current state and future trends. Prostate Int 5(4): 125-129

38. Kim YI, Cheon GJ, Paeng JC, Cho JY, Kwak C, Kang KW et al (2015) Usefulness of MRI-assisted metabolic volumetric parameters provided by simultaneous (18)F-fluorocholine PET/ MRI for primary prostate cancer characterization. Eur J Nucl Med Mol Imaging 42(8):1247-1256

39. Evangelista L, Briganti A, Fanti S, Joniau S, Reske S, Schiavina $R$ et al (2016) New clinical indications for (18)F/(11)C-choline, new tracers for positron emission tomography and a promising hybrid device for prostate cancer staging: a systematic review of the literature. Eur Urol 70(1):161-175

40. Perera M, Papa N, Christidis D, Wetherell D, Hofman MS, Murphy DG et al (2016) Sensitivity, specificity, and predictors of positive (68)Ga-prostate-specific membrane antigen positron emission tomography in advanced prostate cancer: a systematic review and meta-analysis. Eur Urol 70(6):926-937

41. Schuster DM, Nanni C, Fanti S (2016) PET Tracers Beyond FDG in Prostate Cancer. Semin Nucl Med 46(6):507-521

42. Hara T, Kosaka N, Kishi H (1998) PET imaging of prostate cancer using carbon-11-choline. J Nucl Med 39(6):990-995

43. Oyama N, Akino H, Kanamaru H, Suzuki Y, Muramoto S, Yonekura $\mathrm{Y}$ et al (2002) 11C-acetate PET imaging of prostate cancer. J Nucl Med 43(2):181-186

44. Brogsitter C, Zophel K, Kotzerke J (2013) ${ }^{18} \mathrm{~F}$-Choline, ${ }^{11} \mathrm{C}$-choline and ${ }^{11} \mathrm{C}$-acetate PET/CT: comparative analysis for imaging prostate cancer patients. Eur J Nucl Med Mol Imaging 40(Suppl 1):S18-S27

45. Afshar-Oromieh A, Malcher A, Eder M, Eisenhut M, Linhart HG, Hadaschik BA et al (2013) PET imaging with a $\left[{ }^{68} \mathrm{Ga}\right]$ galliumlabelled PSMA ligand for the diagnosis of prostate cancer: biodistribution in humans and first evaluation of tumour lesions. Eur J Nucl Med Mol Imaging 40(4):486-495

46. Gupta SK, Watson T, Denham J, Shakespeare TP, Rutherford $\mathrm{N}$, McLeod N et al (2017) Prostate-specific membrane antigen positron emission tomography-computed tomography for prostate cancer: distribution of disease and implications for radiation therapy planning. Int J Radiat Oncol Biol Phys 99(3):701-709

47. Briganti A, Chun FK, Salonia A, Gallina A, Farina E, Da Pozzo LF et al (2006) Validation of a nomogram predicting the probability of lymph node invasion based on the extent of pelvic lymphadenectomy in patients with clinically localized prostate cancer. BJU Int 98(4):788-793

48. Maurer T, Gschwend JE, Rauscher I, Souvatzoglou M, Haller B, Weirich G et al (2016) Diagnostic efficacy of (68)gallium-PSMA positron emission tomography compared to conventional imaging for lymph node staging of 130 consecutive patients with intermediate to high risk prostate cancer. J Urol 195(5):1436-1443

49. Evangelista L, Cimitan M, Zattoni F, Guttilla A, Zattoni F, Saladini G (2015) Comparison between conventional imaging (abdominal-pelvic computed tomography and bone scan) and [(18)F]choline positron emission tomography/computed tomography imaging for the initial staging of patients with intermediate- to high-risk prostate cancer: a retrospective analysis. Scand J Urol 49(5):345-353

50. Pinaquy JB, De Clermont-Galleran H, Pasticier G, Rigou G, Alberti N, Hindie E et al (2015) Comparative effectiveness of [(18) F]-fluorocholine PET-CT and pelvic MRI with diffusion-weighted imaging for staging in patients with high-risk prostate cancer. Prostate 75(3):323-331

51. Evangelista L, Guttilla A, Zattoni F, Muzzio PC, Zattoni F (2013) Utility of choline positron emission tomography/computed tomography for lymph node involvement identification in intermediateto high-risk prostate cancer: a systematic literature review and meta-analysis. Eur Urol 63(6):1040-1048

52. Budiharto T, Joniau S, Lerut E, Van den Bergh L, Mottaghy F, Deroose CM et al (2011) Prospective evaluation of 11C-choline positron emission tomography/computed tomography and diffusion-weighted magnetic resonance imaging for the nodal staging of prostate cancer with a high risk of lymph node metastases. Eur Urol 60(1):125-130

53. Van den Bergh L, Lerut E, Haustermans K, Deroose CM, Oyen $R$, Isebaert $S$ et al (2015) Final analysis of a prospective trial on functional imaging for nodal staging in patients with prostate cancer at high risk for lymph node involvement. Urol Oncol 33(3):109 e23-109 e31

54. Heck MM, Souvatzoglou M, Retz M, Nawroth R, Kubler H, Maurer T et al (2014) Prospective comparison of computed tomography, diffusion-weighted magnetic resonance imaging and $\left[{ }^{11} \mathrm{C}\right]$ choline positron emission tomography/computed tomography for preoperative lymph node staging in prostate cancer patients. Eur J Nucl Med Mol Imaging 41(4):694-701

55. Poulsen MH, Bouchelouche K, Hoilund-Carlsen PF, Petersen $\mathrm{H}$, Gerke O, Steffansen SI et al $(2012)\left[{ }^{18} \mathrm{~F}\right]$ fluoromethylcholine $(\mathrm{FCH})$ positron emission tomography/computed tomography (PET/CT) for lymph node staging of prostate cancer: a prospective study of 210 patients. BJU Int. 110(11):1666-1671

56. Kjolhede H, Ahlgren G, Almquist H, Liedberg F, Lyttkens $\mathrm{K}$, Ohlsson $\mathrm{T}$ et al (2012) Combined ${ }^{18} \mathrm{~F}$-fluorocholine and ${ }^{18} \mathrm{~F}$-fluoride positron emission tomography/computed tomography imaging for staging of high-risk prostate cancer. BJU Int. 110(10):1501-1506

57. Corfield J, Perera M, Bolton D, Lawrentschuk N (2018) (68)Gaprostate specific membrane antigen (PSMA) positron emission tomography (PET) for primary staging of high-risk prostate cancer: a systematic review. World J Urol. https://doi.org/10.1007/ s00345-018-2182-1

58. Tosoian JJ, Gorin MA, Ross AE, Pienta KJ, Tran PT, Schaeffer EM (2017) Oligometastatic prostate cancer: definitions, clinical outcomes, and treatment considerations. Nat Rev Urol 14(1): $15-25$

59. Gillessen S, Attard G, Beer TM, Beltran H, Bossi A, Bristow $R$ et al (2018) Management of patients with advanced prostate cancer: the report of the Advanced Prostate Cancer Consensus Conference APCCC 2017. Eur Urol 73(2):178-211

60. Sooriakumaran P (2017) Testing radical prostatectomy in men with prostate cancer and oligometastases to the bone: a randomized controlled feasibility trial. BJU Int 120(5B):E8-E20

61. Rexer H (2015) Metastatic, hormone-naive prostate cancer interventional study: multicenter, prospective, randomized study to evaluate the effect of standard drug therapy with or without radical prostatectomy in patients with limited bone metastasized prostate cancer (G-RAMPP - the AUO AP 75/13 study). Urologe A 54(11):1613-1616

62. Sterzing F, Kratochwil C, Fiedler H, Katayama S, Habl G, Kopka $\mathrm{K}$ et al (2016) (68)Ga-PSMA-11 PET/CT: a new technique with high potential for the radiotherapeutic management of prostate cancer patients. Eur J Nucl Med Mol Imaging 43(1):34-41

63. Denham JW, Joseph D, Lamb DS, Spry NA, Duchesne G, Matthews J et al (2014) Short-term androgen suppression and radiotherapy versus intermediate-term androgen suppression and radiotherapy, with or without zoledronic acid, in men with locally advanced prostate cancer (TROG 03.04 RADAR): an 
open-label, randomised, phase 3 factorial trial. Lancet Oncol 15(10):1076-1089

64. Bolla M, Van Tienhoven G, Warde P, Dubois JB, Mirimanoff RO, Storme G et al (2010) External irradiation with or without long-term androgen suppression for prostate cancer with high metastatic risk: 10-year results of an EORTC randomised study. Lancet Oncol 11(11):1066-1073

65. Borgmann H, Wolm JH, Vallo S, Mager R, Huber J, Breyer J et al (2017) Prostate cancer on the web-expedient tool for patients' decision-making? J Cancer Educ 32(1):135-140

66. Kyriakopoulos CE, Chen YH, Carducci MA, Liu G, Jarrard DF, Hahn NM et al (2018) Chemohormonal therapy in metastatic hormone-sensitive prostate cancer: long-term survival analysis of the randomized phase III E3805 CHAARTED trial. J Clin Oncol. https://doi.org/10.1200/JCO.2017.75.3657

67. Sweeney CJ, Chen YH, Carducci M, Liu G, Jarrard DF, Eisenberger $\mathrm{M}$ et al (2015) Chemohormonal therapy in metastatic hormone-sensitive prostate cancer. N Engl J Med 373(8):737-746

68. Fizazi K, Tran N, Fein L, Matsubara N, Rodriguez-Antolin A, Alekseev BY et al (2017) Abiraterone plus prednisone in metastatic, castration-sensitive prostate cancer. N Engl J Med 377(4):352-360 